The following scientific article was officially published in the journal Computers in Biology and Medicine, published by Elsevier. This article's citation is as follows:

Assi, Kondo Claude, Hubert Labelle, and Farida Cheriet. "Statistical model based 3D shape prediction of postoperative trunks for non-invasive scoliosis surgery planning." Computers in Biology and Medicine, Vol. 48, (2014): pp. 85-93.

doi: $10.1016 /$ j.compbiomed.2014.02.015

The manuscript, as accepted by the publisher, is reproduced here, as it appears in the first author's Ph.D. thesis, entitled Modélisation physique des tissus mous du tronc scoliotique pour la simulation de l'apparence post-chirurgicale. The thesis citation is as follows:

Assi, Kondo Claude. "Modélisation physique des tissus mous du tronc scoliotique pour la simulation de l'apparence post-chirurgicale." PhD diss., École Polytechnique de Montréal, 2014.

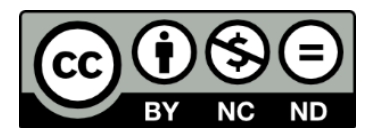

Kondo Claude Assi, 2014

(C) 2014 Kondo Claude Assi. This work is licensed under the Creative Commons AttributionNonCommercial-NoDerivatives 4.0 International License. To view a copy of this license, visit:

http://creativecommons.org/licenses/by-nc-nd/4.0/ 


\title{
CHAPITRE 5
}

\section{ARTICLE 2: STATISTICAL MODEL BASED 3D SHAPE PREDICTION OF POSTOPERATIVE TRUNKS FOR NON-INVASIVE SCOLIOSIS SURGERY PLANNING}

\author{
K. C. Assi ${ }^{a, b, *}$, H. Labelle ${ }^{b}$, F. Cheriet ${ }^{a, b}$ \\ (a) École Polytechnique de Montréal, P.O. Box 6097, Succursale Centre-ville, \\ Montréal, Québec, Canada H3C 3 A7 \\ (b) Sainte-Justine Hospital Research Center, 3175 Côte-Sainte-Catherine, \\ Montréal, Québec, Canada H3T 1C5
}

\subsection{Abstract}

One of the major concern of scoliosis patients undergoing surgical treatment is the aesthetic aspect of the surgery outcome. It would be useful to predict the postoperative appearance of the patient trunk in the course of a surgery planning process in order to take into account the expectations of the patient. In this paper, we propose to use the least squares support vector regression for the prediction of the postoperative trunk 3D shape after spine surgery for adolescent idiopathic scoliosis. Five dimensionality reduction techniques used in conjunction with the support vector machine are compared. The methods are evaluated in terms of their accuracy, based on the leave-one-out cross-validation performed on a database of 141 cases. The results indicate that the $3 \mathrm{D}$ shape predictions using a dimensionality reduction obtained by simultaneous decomposition of the predictors and response variables have the best accuracy.

\subsection{Introduction}

Adolescent idiopathic scoliosis (AIS) is a three-dimensional deformation of the spine and rib cage, with a prevalence of approximately $3 \%$ in the general population. Depending on the severity of the trunk deformity, a spine correction surgery may be required. Among patients with AIS, 9\% are treated by brace and one in a thousand needs surgery using posterior spinal instrumentation and fusion to correct the deformity. Neglecting to proceed for a surgical treatment may lead to functional complications such as postural, cardiac or pulmonary problems.

One of the main concern of scoliotic patients undergoing surgery is the postoperative external appearance of the trunk, i.e, the aesthetical aspect [17]. Assessment by surgeons 
of scoliosis surgery outcomes are not necessarily correlated with patient satisfaction and vary with the observer [89]. Therefore, it would be important to be able to rigorously and quantitatively predict the outcome of the surgery in a surgery planning process. This will allow the surgeon to take into account the expectations of the patients.

Information technology in medicine is assisting physicians and clinicians in their practice by providing computer based data management and processing tools as an aid in medical decision making. Recently, Devedzic et al. [90] developed a web-based information system, ScolioMedIS, to help health professionals monitor, manage and use clinical data from scoliosis patients. ScolioMedIS [90] uses parameterized 3D anatomical models of the spine for the quantitative assessment of the deformity. By reducing the number of required radiographs, the system allows to minimize the amount of radiation exposure. One of the turning points in scoliosis research was the emergence, in the last two decades, of new techniques for the human trunk surface topography data acquisition and processing, mostly based on optical range image 3D technologies. The aim was to replace radiographic evaluation of scoliosis with systems free from ionizing radiation. Pazos et al. [91] developed a non-invasive technique with a four digital cameras using a projected structured light for the acquisition and 3D reconstruction of the whole trunk. This optical 3D digitizing system is used for clinical assessment of the trunk external asymmetry. Shannon [92] developed a system using video based motion capture technology allowing the simultaneous acquisition of multiple samples of back surface shape and the locations of bony landmarks. This system leads to a quantitative and reliable analysis of the trunk aesthetics. Some researchers have proposed, in the past recent years, various efficient 3D surface analysis combined with different trunk landmarks identification and selection techniques to detect spinal and torso deformities, leading to different trunk surface characterization schemes for scoliosis diagnosis. For example, using structured splines models of the trunk surface, Ajemba et al. [93] proposed a method of characterizing the torso shape deformity associated with scoliosis by both its type and severity. Seoud et al. [69] used some trunk surface topography analysis techniques to obtain the prediction of scoliosis curve types. These previous works succeeded in fulfilling their purpose but did not address the surgical treatment outcome prediction problem. The present paper contribution builds upon the above cited works in the analysis of the trunk surface topography for the management of scoliosis and proposes a method to predict the postoperative trunk 3D shape.

Machine learning prediction techniques, particularly support vector machines (SVM), have been increasingly and successfully applied to a wide variety of biological and medical engineering problems such as electroencephalography (EEG) signals analysis [64, 94], electromyography (EMG) signals analysis for neuromuscular disorder detection [95, brain glioma [65], heart disease diagnosis [96], macroinvertebrate image classification [97], and pro- 
tein recognition [98], to cite but a few examples. In the case of scolosis studies, one important question that was addressed by different research groups, during the last few years, is about relating the trunk surface with the configuration of the spine. Bergeron et al. 66] have investigated the correlation between the surface topography and the spinal curve, using support vector regression. Jaremko et al. [67] have used neural-network approaches to study and estimate Cobb angle from the trunk external surface asymmetry measurements. Ramirez et al. 68] have used support vector machine to assess the severity of the spine deformity from surface topography data. Although these works find applications in the follow-up or diagnosis of scoliosis patients, they were essentially limited to preoperative trunk shapes. These studies do not address the question of correlating the preoperative shape with the postoperative shape. Finding a solution to such a question would have important applications in scoliosis surgery planning.

Very few works have been done on the quantitative relationship between the preoperative and the postoperative trunk surfaces. Simulation of the postoperative bone structure have been conducted in [4]. But the study was limited only to the spine configuration. To the best of our knowledge, the first work on the simulation of the postoperative trunk surface in scoliosis surgery are the preliminary results reported in [29] by our research group. The approach is based on a particle system model of the soft-tissue of the trunk. This simulator has however some limitations. In particular, some aspect of the preprocessing requires the output of a postoperative bone structures simulator [4] as a starting point. The approach taken in the present work, is through shape prediction based on support vector machines, and complements the simulator in [29]. The output of the study would provide to the simulator an initial shape estimate to be used in the personalized trunk surface simulation. Our approach takes into account information contained in a database of scoliotic trunk 3D shapes. To the best of our knowledge, this is the first study of statistical based prediction of the postoperative trunk 3D shape after spine surgery for AIS.

The main idea of statistical model-based shape prediction is to find statistically plausible parameter values of the model, given the available patient data and some fitting criteria. Most statistical machine learning techniques may not be effective for high-dimensional data. Due to the curse of dimensionality, the accuracy and efficiency of most prediction algorithms degrade rapidly as the data dimension increases. Medical images and anatomical shapes data usually involves a large number of samples points and correlated features. Thus, it is a prerequisite to reduce the data dimension so that the prediction methods can be applied to the reduced data sets. For this reason, a typical prediction procedure may consist in four steps: (i) feature extraction, (ii) feature dimensionality reduction, (iii) regression function learning, and (iv) prediction [99]. The choice of support vector machine for regression as a learning 
method was motivated by its strong generalizability capability. Dimensionality reduction is the process of mapping high-dimensional feature vectors onto lower-dimensional vectors while maintaining sufficient information to reproduce most of the variability of the original data set. Principal components analysis (PCA) [100] is by far the most popular unsupervised linear technique for dimension reduction. PCA constructs a low-dimensional representation of the data that describes as much of the variance in the data as possible. However, its effectiveness is limited by its global linearity. Kernel PCA (KPCA) [101] is the reformulation of the traditional linear PCA in a high-dimensional space that is constructed using a kernel function. KPCA computes the principal eigenvectors of the kernel matrix, rather than those of the covariance matrix. However, an important weakness of KPCA is that the size of the kernel matrix is proportional to the square of the number of instances in the data set. The Locality preserving projections (LPP) method [102] is a technique that aims at combining the benefits of linear techniques and local nonlinear techniques for dimensionality reduction by finding a linear mapping that minimizes the cost function of Laplacian eigenmaps. In contrast to PCA, LPP method is capable of successfully identifying complex data manifolds such as the Swiss roll. Neighborhood preserving embedding (NPE) [103] is similar to LPP. However, NPE proceeds by minimizing the cost function of a local nonlinear technique for dimensionality reduction under the constraint that the mapping from the high dimensional to the low-dimensional data representation is linear. Partial Least Squares (PLS) [104, 105] is a technique that generalizes and combines features from principal component analysis and multiple regression. It is particularly useful when one needs to predict a set of dependent variables from a very large set of independent predictor variables. The underlying assumption of all PLS methods is that the observed data is generated by a process which is driven by a small number of latent variables. In its general form, PLS creates orthogonal latent vectors by using the existing correlations between the sets of input and output variables while also keeping most of the variance of both sets. Due to its capability of effectively decreasing the correlation between the input influence factors, the PLS method has proven to be useful in situations where the number of observed variables is much greater than the number of observations and high multicollinearity among the variables exists. These methods were mainly used in the context of classification problems.

The aim of this paper is to compare different dimensionality reduction techniques for a statistical model based shape prediction of the trunk. We report the results obtained using a multivariate statistical model of the trunk feature curve. Five dimensionality reduction techniques such as principal component analysis (PCA), Kernel principal component analysis (KPCA), partial least squares (PLS), locality preserving projection (LPP) and neighborhood preserving embedding (NPE) are considered and compared on a database of 141 scoliosis 
patients trunk shapes. The prediction results are evaluated using the leave-one-out crossvalidation.

\subsection{Materials and Methods}

The methods for the trunk 3D shape prediction are described in this section. All the evaluations were made on real clinical datasets obtained using a non-invasive medical imaging system.

\subsubsection{Data acquisition of the trunk surface}

The data were acquired at the Sainte-Justine Hospital Research Center, and consist of 141 samples. Trunk data were acquired from patients with AIS of age between 11 and 18 years old, who have undergone spine surgery. The acquisition protocol was described by Pazos et al. [106, 88] and is summarized in this section. The surface geometry of the trunk is acquired using a calibrated system composed of four 3D optical digitizers (InSpeck Inc., Montreal, Canada), each assembled with a CCD camera and a structured light projector, placed around the patient (Figure 5.1). The acquisition process, identical for each scanner, consists in projecting and capturing four fringe patterns deformed by the trunk's external shape. The system then computes, by triangulation, the depth of each surface point relative to the reference plane of the digitizer. A fifth image, captured without fringes, defines the texture data mapped on the surface. The entire trunk geometry is obtained by registering

and merging the partial surfaces obtained by each digitizer. This process takes 4-6 seconds with the patient standing still in the upright position, arms slightly abducted to prevent occluded areas in the field of view of the lateral scanners. The resulting mesh contains $40 \mathrm{k}-70 \mathrm{k}$ vertices, depending on the patient's height and a previous study [88] of the system demonstrated a reconstruction accuracy of $1.4 \mathrm{~mm}$ over the whole torso of a mannequin. Before the surface acquisition, visible landmarks are drawn on the patient's skin. The accuracy of the $3 \mathrm{D}$ position of the identified landmarks was evaluated at $2.1 \pm 1.5 \mathrm{~mm}$ [20]. The homologous landmarks coordinates for each geometry are latter used for the alignement of all the surfaces.

\subsubsection{Feature curves representation of the trunk}

The trunk surface may be viewed and represented as a finite collection of 3D curves. For the purpose of this work, we are interested in characteristic anatomical curves on the trunk external surface. One of the most visible features of the trunk external surface is the socalled back valley. Figure 5.2 shows the back valley curve along the spinous processes of a scoliotic patient in the preoperative and the postoperative status. The shape of this feature 


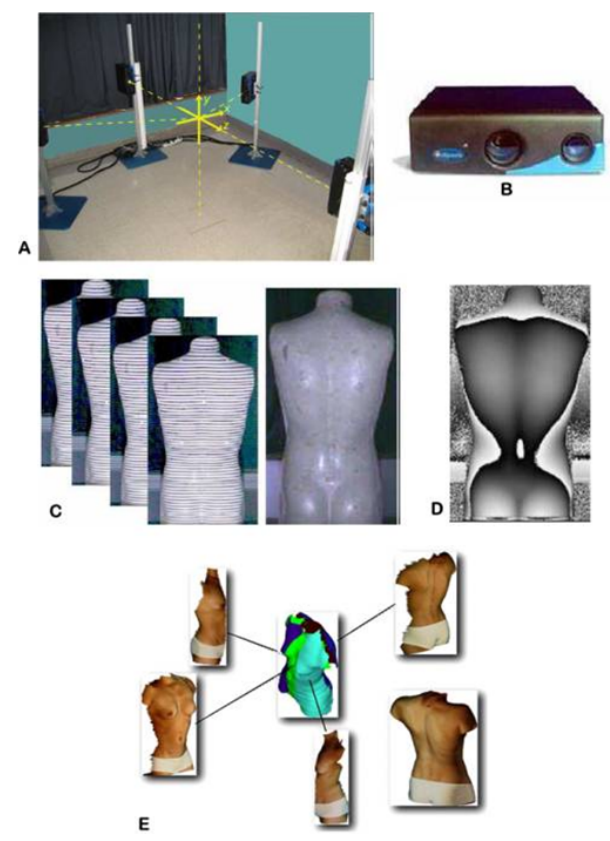

Figure 5.1 Trunk topography measurement and reconstruction. (A) Experimental set-up of four optical digitizers. (B) Example of a Capturor 3D optical digitizer, consisting of a CCD camera coupled with a structured light projector. (C) Set of four fringe images, each offset by $\frac{1}{4}$ phase, projected by a digitizer onto the back of a mannequin. A fifth image provided the surface texture. (D) Resulting phase image from the four fringe images; surface reconstruction uses the interferometry principle combined with active triangulation. (E) The process of registering and merging the partial surfaces from the different digitizers produces the complete trunk surface
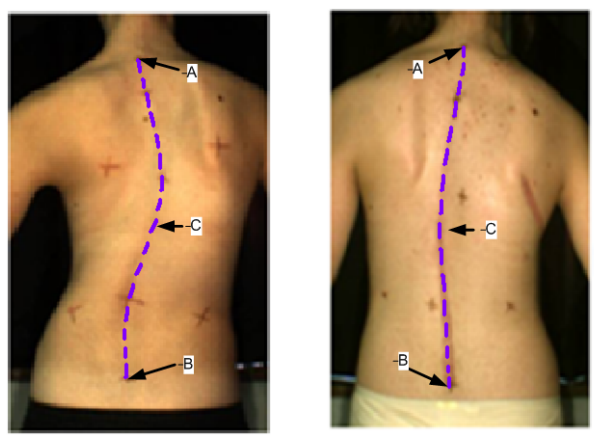

Figure 5.2 Feature curves (back valley) of the trunk surface. Left: preoperative surface. Right: postoperative surface. The curve top and bottom endpoints $A$ and $B$ correspond to the spinous process of $C 7$ and the spinous process of $L 5$, respectively. $C$ indicates the curve.

curve almost follows that of the spine. Due to the surgery procedure, the curve shape has changed from preoperative to postoperative state. The upper and lower end points $(A$ and $B)$ are anatomical landmarks corresponding to the spinous process of $C 7$ (seventh 
cervical vertebra) and the spinous process of $L 5$ (the fifth lumbar vertebra), respectively. A positioning system ensured standard posture before data acquisition. The results of the raw feature curve extraction is a polygonal line consisting of up to 75 three-dimensional coordinates samples from the back surface located along the back valley (Figure 5.3). An initial processing of the data provides a similar basis for comparison among all patients. To begin, four surface markers appearing on the trunk datasets were used to translate the point coordinates to a common 3D Euclidean space, having as origin the spinous process of $L 5$ vertebra. The $x$-axis was oriented towards the front of the patient, the $y$-axis towards the
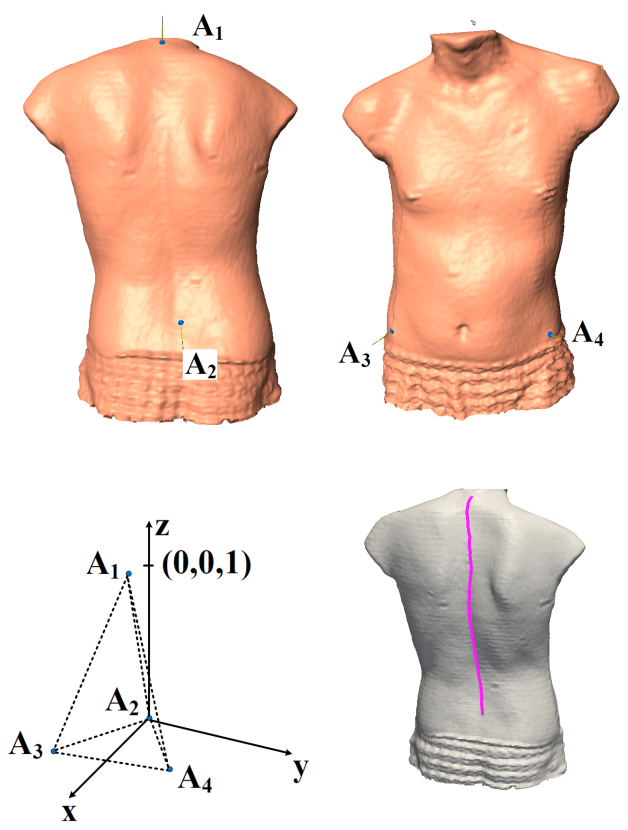

Figure 5.3 Anatomical landmarks for the normalization of the dataset. Four landmarks $\left(A_{1}\right)$ PV,$\left(A_{2}\right)$ MPSIS , $\left(A_{3}\right)$ LASIS, and $\left(A_{4}\right)$ RASIS are considered to define a unified coordinate systems. The origin is set at $A_{2}$. The $z$-axis is perpendicaular to the $\left(A_{2} A_{3} A_{4}\right)$ plane. The $y$-axis is parallel to ASIS and oriented to the left side of the patient. The $x$-axis is oriented to the front of the patient.

patient left (parallel to the ASIS axis). That space was scaled to the unit length of the distance between the endpoints $A_{1}$ and $A_{2}$ (Figure 5.3), after which the curve was rotated about the origin such that the curve upper end point $A_{1}$ coincide with the point $(0,0,1)$. The feature curves were represented explicitly as a finite set of corresponding points uniformly sampled from a parameterized function $\mathbf{f}=\mathbf{f}(t), \mathbf{f}:[0,1] \rightarrow \mathbb{R}^{3}$, fitting the initial points. 


\subsubsection{Statistical framework}

The input and response variables $\mathbf{x}_{i}$ and $\mathbf{y}_{i}$ for the $i$-th observation $(i=1, \ldots, n)$ are as follow. The variables $\mathbf{x}_{i}$ are obtained from the positions of $N$ points uniformly sampled from the feature curve of the preoperative trunk shape, while the variables $\mathbf{y}_{i}$ are points sampled from the postoperative trunk shape:

$$
\begin{aligned}
& \mathbf{x}_{i}=\left[\begin{array}{lllllll}
\mathbf{P}_{1}^{x} & \mathbf{P}_{1}^{y} & \mathbf{P}_{1}^{z} & \ldots & \mathbf{P}_{N}^{x} & \mathbf{P}_{N}^{y} & \mathbf{P}_{N}^{z}
\end{array}\right],
\end{aligned}
$$

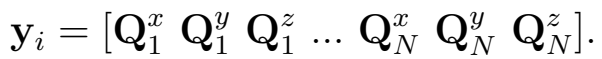

The data are scaled and centered according to the procedure described in Section 5.3.2. The data matrices $\mathbf{X}$ and $\mathbf{Y}$, of size $n \times 3 N$, contain the variables corresponding to the $n$ preprocessed samples $\mathbf{x}_{i}$ and $\mathbf{y}_{i}$ of the dataset. The aim is to predict the shape of the postoperative curve 3D shape from the preoperative curve shape. The prediction of the postoperative 3D trunk shape $\mathbf{y}$ from the preoperative trunk shape $\mathbf{x}$ can be performed using the Least Square Support Vector Machine (LS-SVM) [107], in conjunction with a dimensionality reduction of the data prior to the LS-SVM regression. In this study, five dimensionality reduction methods are investigated, namely the Principal Component Analysis (PCA), the Kernel Principal Component Analysis (KPCA), the Partial Least Squares (PLS), the Locality Preserving Projection (LPP) and the Neighborhood Preserving Embedding (NPE). In the remanining of this paper, we adopt the following notations. The dot on top of a variable indicates that it is centered: $\dot{v}=v-\mu_{V}, \mu_{V}$ being the sample mean.

\section{Principal Component Analysis}

The PCA approach [100 finds directions $\mathbf{u}_{i}$ where the the variance of $\dot{\mathbf{X}}$ (or similarly $\left.\mathbf{u}_{i}^{T} \dot{\mathbf{X}} \dot{\mathbf{X}}^{T} \mathbf{u}_{i}\right)$ is maximum. For that, PCA solves the eigenvalue problem:

$$
\dot{\mathbf{X}}^{T} \dot{\mathbf{X}} \mathbf{u}=\lambda \mathbf{u}
$$

The reduced dataset is obtained by linear projection: $\tilde{\mathbf{X}}=\dot{\mathbf{X}} \mathbf{T}$, where $\mathbf{T}$ is formed by the eigenvectors of the centered covariance matrix. 


\section{Kernel Principal Component Analysis}

The Kernel PCA dimensionality reduction [101] computes the reduced dataset by the projection of the (training points) $\left\{\mathbf{x}_{i}\right\}_{i=1}^{n}$ as

$$
\mathbf{P}=\tilde{\mathbf{U}} \tilde{\Lambda}^{1 / 2}
$$

where the columns of $\tilde{\mathbf{U}}$ are created by the eigenvectors $\left\{\tilde{\mathbf{u}}^{i}\right\}_{i=1}^{n}$, and $\tilde{\boldsymbol{\Lambda}}$ is a diagonal matrix $\operatorname{diag}\left(\tilde{\lambda}_{1}, \ldots, \tilde{\lambda}_{n}\right)$ are obtained by solving the eigenvalue problem

$$
\mathbf{K} \tilde{\mathbf{u}}=\tilde{\lambda} \tilde{\mathbf{u}}
$$

with $\mathbf{K}=\boldsymbol{\Phi} \boldsymbol{\Phi}^{T}$ the $(n \times n)$ matrix associated to the $(n \times n)$ matrix of the centered nonlinear mappings of the input variables $\left\{\mathbf{x}_{i}\right\}_{i=1}^{n} \in \mathbb{R}^{n}$.

\section{Partial Least Squares}

The PLS dimensionality reduction method [104, 105] finds pairs of direction $\mathbf{u}_{i}, \mathbf{v}_{i}$, so that

$$
\rho_{i}=\mathbf{u}_{i}^{T} \dot{\mathbf{X}} \dot{\mathbf{Y}}^{T} \mathbf{v}_{i}
$$

is maximal, with the constraint that both $\mathbf{U}_{r}=\left[\mathbf{u}_{1}, \ldots, \mathbf{u}_{r}\right]$ and $\mathbf{V}_{r}=\left[\mathbf{v}_{1}, \ldots, \mathbf{v}_{r}\right]$ form orthonormal bases. Those directions are obtained by solving the eigenproblem:

$$
\begin{aligned}
\mathbf{S}_{\mathbf{x y}} \mathbf{S}_{\mathbf{y x}} \mathbf{u}_{i} & =\rho_{i}^{2} \mathbf{u}_{i} \\
\mathbf{S}_{\mathbf{y x}} \mathbf{S}_{\mathbf{x y}} \mathbf{v}_{i} & =\rho_{i}^{2} \mathbf{v}_{i}
\end{aligned}
$$

where $\mathbf{S}_{\mathbf{x y}}, \mathbf{S}_{\mathbf{y x}}$ are the sample covariance matrices of $X$ and $Y$, and $Y$ and $X$, respectively. The scores $\mathbf{T}_{r}$ are given by the projection:

$$
\mathbf{T}_{r}=\dot{\mathbf{X}}^{T} \mathbf{U}_{r}
$$

\section{Locality Preserving Projection}

The Locality Preserving Projection (LPP) method [102] is a linear approximation of the nonlinear Laplacian EigenMap. It computes the mapped dataset $\tilde{\mathbf{X}}=\dot{\mathbf{X}} \mathbf{A}$, where $\mathbf{A}$ is composed by the eigenvectors of the generalized eigenvector problem

$$
\dot{\mathbf{X}}^{T} \mathbf{L} \dot{\mathbf{X}} \mathbf{v}=\lambda \dot{\mathbf{X}}^{T} \mathbf{M} \dot{\mathbf{X}} \mathbf{v}
$$


in which $\mathbf{L}$ is the graph Laplacean and $\mathbf{M}$ the degree matrix of the graph whose weights are given by $w_{i j}=e^{-\frac{\left\|\mathbf{x}_{i}-\mathbf{x}_{j}\right\|^{2}}{2 \sigma^{2}}}$.

\section{Neighborhood Preserving Embedding}

The NPE [103] projects the data with the projection $\tilde{\mathbf{X}}=\dot{\mathbf{X}} \mathbf{B}$ where the linear mapping $\mathbf{B}$ is formed by the eigenvectors associated to the first smallest nonzero eigenvalues of the generalized eigenproblem

$$
\dot{\mathbf{X}}^{T}(\mathbf{I}-\mathbf{W})^{T}(\mathbf{I}-\mathbf{W}) \dot{\mathbf{X}} \mathbf{v}=\lambda \dot{\mathbf{X}}^{T} \dot{\mathbf{X}} \mathbf{v}
$$

where $\mathbf{I}$ represents the $n \times n$ identity matrix, and $\mathbf{W}$ the adjacency matrix of the neighborhood graph.

\subsubsection{Least Squares Support Vector Regression}

The least squares support vector machine (LS-SVM) for regression [107] is used for the prediction of the postoperative trunk 3D curve shapes. Given the dataset $\left\{\left(\mathbf{x}_{i}, y_{i}\right)_{i=1}^{n}\right\}$, we are interested in estimating a model of the form

$$
y=\mathbf{w}^{T} \phi(\mathbf{x})+\mathbf{b},
$$

where $\mathbf{x} \in \mathbb{R}^{n}, y \in \mathbb{R}$ and $\phi():. \mathbb{R}^{n} \rightarrow \mathbb{R}^{n_{h}}$ is the mapping to a high dimensional feature space. LS-SVM solves the optimization problem:

$$
\begin{aligned}
& \min _{\mathbf{w}, \mathbf{b}, \mathbf{e}} \frac{1}{2} \mathbf{w}^{T} \mathbf{w}+\gamma \frac{1}{2} \sum_{i=1}^{N} e_{i}^{2} \\
& \text { subject to } y_{i}=\mathbf{w}^{T} \phi\left(\mathbf{x}_{i}\right)+\mathbf{b}+e_{i}, \quad i=1, \ldots, N,
\end{aligned}
$$

where $\gamma>0$ is a regularization parameter. The associated Lagrangian is:

$$
L_{\alpha}(\mathbf{w}, \mathbf{b}, \mathbf{e})=\frac{1}{2} \mathbf{w}^{T} \mathbf{w}+\gamma \frac{1}{2} \sum_{i=1}^{N} e_{i}^{2}-\sum_{i=1}^{N} \alpha_{i}\left(\mathbf{w}^{T} \phi\left(\mathbf{x}_{i}\right)+\mathbf{b}+e_{i}-y_{i}\right) .
$$

The Karush-Kuhn-Tucker (KKT) conditions for optimality lead to the linear system

$$
\left[\begin{array}{cc}
0 & \mathbf{1}_{N}^{T} \\
\mathbf{1}_{N} & \mathbf{Z}^{T} \mathbf{Z}+\frac{1}{\gamma} \mathbf{I}
\end{array}\right]\left[\begin{array}{l}
\mathbf{b} \\
\alpha
\end{array}\right]=\left[\begin{array}{l}
\mathbf{0} \\
y
\end{array}\right]
$$


where

$$
\mathbf{Z}^{T}=\left[\begin{array}{c}
\phi\left(\mathbf{x}_{1}\right)^{T} y_{1} \\
\phi\left(\mathbf{x}_{2}\right)^{T} y_{2} \\
\cdots \\
\phi\left(\mathbf{x}_{N}\right)^{T} y_{N}
\end{array}\right]
$$

The resulting LS-SVM model is given by $\hat{y}(\mathbf{x})=\sum_{i=1}^{N} \alpha_{i} K\left(\mathbf{x}, \mathbf{x}_{i}\right)+\mathbf{b}$, where $K\left(\mathbf{x}_{i}, \mathbf{x}_{j}\right)=$ $\phi\left(\mathbf{x}_{i}\right)^{T} \phi\left(\mathbf{x}_{j}\right)$ is the kernel function.

\subsection{Results}

A cohort of 141 scoliotic shapes data, from teenagers aged between 11 - 18 years old, are considered. A few samples of the data set obtained by the procedure described in Section 5.3 .2
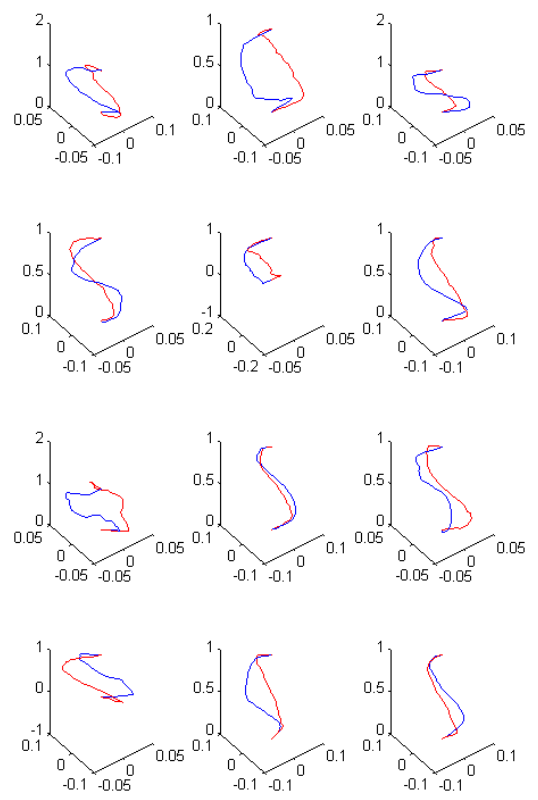

Figure 5.4 Samples of scaled data of the preoperative curve (blue) and postoperative curve (red). The data have been scaled to unit length of the distance between the curve endpoints.

are presented in Figure 5.4, where the preoperative back curves (blue) are shown along with the postoperative curves (red). 


\subsubsection{Validation}

The dimensionality reduction methods combined to the LS-SVM regression for the trunk 3D shape prediction are evaluated using the leave-one-out cross-validation procedure. A sample of the data is, in turn, removed from the dataset and used as a test point. The statistical model is trained by using the remaining samples. The prediction procedure is then applied to the test sample. Each sample point is composed of a pair (preoperative and postoperative shapes). The predicted curve can then be compared with the actual postoperative feature curve and a shape prediction error can be computed. Quantitative prediction errors are obtained by computing the distances between corresponding points on the predicted back feature curve and the measured back curve of the postoperative test case. To evaluate our results, we compute the prediction error in terms of the normalized root mean square prediction error, which has the advantage of allowing prediction error to be measured on the same scale for all observations.

\subsubsection{Results}

The leave-one-out cross validation procedure described in Sections 5.4.1 was conducted on a database of $n=141$ cases. The prediction errors distribution for the input data, reduced to (25) components, are presented in Figure 5.5 for the dimensionality reduction methods presented in Section 5.3.3. The mean prediction errors are $0.0145( \pm 0.0067)$ for the classical

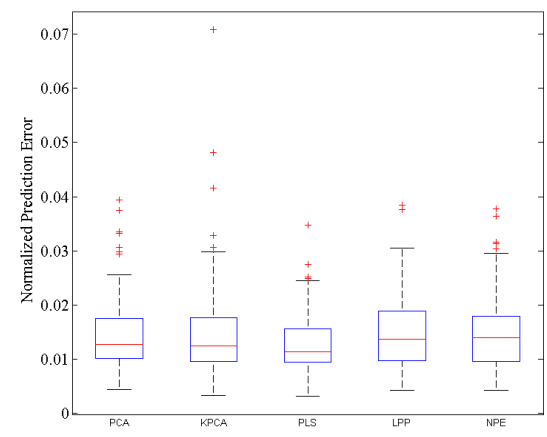

Figure 5.5 Comparison of the effect of dimensionality reduction methods on the trunk 3D shape prediction using LS-SVR regression. Summary of prediction errors for different dimensionality reduction methods: Box plots of mean prediction errors. The performances are compared in normalized root mean squared error (NRMS) terms. From left to right, Principal Component Analysis, Kernel Principal Component Analysis, Partial Least Squares, Locality Preserving Projection, and Neighborhood Preserving Embedding.

PCA method, 0.0148 $( \pm 0.0086)$ for the KPCA, 0.0126( \pm 0.0052$)$ for the PLS, $0.0149( \pm 0.0071)$ 
for the LPP and 0.0146( \pm 0.0065$)$ for NPE. It appears that the PLS has the best results, on average, for the prediction. From these results it can be seen that the PLS-LSSVM regression has better performances and smaller standard deviations compared to the other methods. An example of the prediction with the PLS-LSSVM regression of postoperative 3D shape is presented in Figure 5.6, where the three-dimensional predicted postoperative curve is overlayed on the postoperative trunk surface mesh, along with the preoperative curve and the actual postoperative back valley curve. As one can notice the 3D shape predicted by the

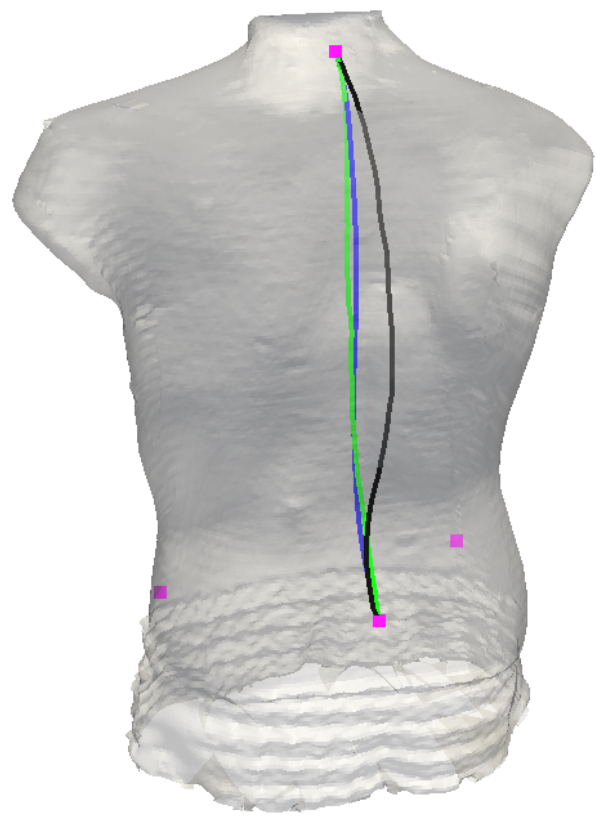

Figure 5.6 Three-dimensional visualization of the curve shape for an example patient. Overlay of the predicted curves on the actual postoperative trunk surface. preoperative (black), actual postoperative (green) and predicted postoperative with PLS-LSSVR (blue).

PLS-LSSVM regression is close to the actual postoperative shape.

\subsection{Discussion}

Since the back valley line is an open curve with well identified endpoints, the use of an uniform sampling to define the corresponding points is reasonable. This procedure allows to solve the point correspondence problem which is necessary for the statistical model. The study, conducted on a database of 141 samples pairs, uses a finite set of corresponding points on the trunk feature curves.

The different dimensionality reduction methods appears to perform in a similar way. Unexpectedly, however, the kernel PCA appears to produce less accurate postoperative 3D 
shape predictions compared to the classical PCA. This may be due to the difficulties related to the tuning parameters selection in the higher dimension space.

However, the PLS method in particular manages to capture well the relations between the preoperative shape and the postoperative shape compared to the other methods. The PLS exploits the correlation between the input space data and the response data, which explain the better performance obtained on the predictions. It performs a simultaneous decomposition of the input and response with the constraint that these components explain as much as possible of the covariance between input and response data.

The LPP and NPE methods proved to generate results very similar to the PCA and KPCA approaches. It is not surprising to see that the LPP and NPE methods perform on average as good as the classical PCA, since the neighborhood information is exploited in the prediction model. These results support the idea that closer points in the input shape space are expected to behave similarly in the response shape space. However, we believe that a procedure for learning the appropriate distance metric in the trunk shape spaces would improve the accuracy performance of the predictions based on LPP and NPE.

\subsection{Conclusion}

To date, the statistical approaches to the trunk 3D shapes analysis for the clinical management of scoliosis were mainly aimed at the scoliosis curve types detection and classification. To the best of our knowledge, there exists no previous research reporting on the statistical prediction of the trunk postoperative shape based on the preoperative data for surgically treated patients with scoliosis. In the present paper, we have contributed to the analysis of the trunk surface topography by proposing novel approaches to predict the postoperative trunk 3D shapes of scoliosis patients. The approaches consist in the combination of dimensionality reduction techniques with machine learning based prediction methods. This is the first work on the subject.

The prediction of the postoperative $3 D$ shapes of scoliotic trunks, stated as a regression problem of predicting the positions of a finite set of points of the trunk feature curves knowing their initial positions in preoperative state, has been approached using least squares support vector machine. Different dimensionality reduction techniques in conjunction with the support vector regression have been compared. The accuracy of the prediction errors has been analyzed using the leave-one-out cross-validation. Although, the methods performed similarly on average, the PLS dimensionality reduction appears to provide better results. This study may however have some limitations, in that in particular, anthropometric predictor variables (such as gender, age, body mass index) were not explicitly included. 
As a future work, additional landmark-curves will be considered in order to predict the whole trunk surface. Some aspects of the present work will be useful as a complementary data supplier to the framework of the personalized physically-based trunk soft-tissue modeling used for surgery simulation [29] by providing an initial guess of the simulated trunk.

\section{Conflict of interest statement}

The authors declare that they have no conflict of interest.

\section{Acknowledgments}

This research was funded by the Natural Sciences and Engineering Research Council of Canada. The equipment was financed by the Canadian Foundation for Innovation. Au-

thor 1 was supported, in part, by the Fondation Sainte-Justine and Fondation des Étoiles foundations of Sainte-Justine University Hospital Center. 


\section{RÉFÉRENCES}

[1] S. Kadoury, F. Cheriet, C. Laporte, and H. Labelle. A versatile 3D reconstruction system of the spine and pelvis for clinical assessment of spinal deformities. Medical and Biological Engineering and Computing, 45(6) :591-602, 2007.

[2] W. Mollemans, F. Schutyser, N. Nadjmi, F. Maes, and P. Suetens. Predicting soft tissue deformations for maxillofacial surgery planning system : from computational strategies to a complete clinical validation. Medical Image Analysis, 11(3) :282-301, 2007.

[3] C.-E. Aubin, H. Labelle, and O. C. Ciolofan. Variability of spinal instrumentation configurations in adolescent idiopathic scoliosis. European Spine Journal, 16(1) :57-64, 2007.

[4] C.-E. Aubin, H. Labelle, C. Chevrefils, G. Desroches, J. Clin, and A. B. Eng. Preoperative planning simulator for spinal deformity surgeries. Spine, 33(20) :2143-2152, 2008.

[5] V. J. Raso, E. Lou, D. L. Hill, J. K. Mahood, M. J. Moreau, and N. G. Durdle. Trunk distorsion in adolescent idiopathic scoliosis. J.Pediatr. Orthop., 18 :222-226, 1998.

[6] C. Denoel, M. F. I. Aguirre, G. Bianco, P. H. Mahaudens, R. Vanwijck, S. Garson, R. Sinna, and A. Debrun. Idiopathic scoliosis and breast asymmetry. Journal of Plastic Reconstructive and Aesthetic Surgery, 62 :1303-1308, 2009.

[7] T. G. Lowe, M. Edgar, J. Y. Margulies, N. H. Miller, V. J. Raso, K. A. Reinker, and C. H. Rivard. Etiology of idiopathic scoliosis : current trends in research. J Bone Joint Surg Am, 82-A :1157-1168, 2000.

[8] E. J. Rogala, D. S. Drummond, and J. Gurr. Scoliosis : incidence and natural history. a prospective epidemiological study. J Bone Joint Surg Am, 60 :173-176, 1978.

[9] J.R. Cobb. Outline for the study of scoliosis. Am. Acad. Orthop. Surg. Instruct. Lect., 5 :261-275, 1948.

[10] S. Negrini. Bracing adolescent idiopathic scoliosis today. Disabil Rehabil Assist Technol, $3: 107-111,2008$.

[11] R.R. Betz and H. Shufflebarger. Anterior versus posterior instrumentation for the correction of thoracic idiopathic scoliosis. Spine, 26(9) :1095-1100, 2001. 
[12] P. Papin, H. Labelle, S. Delorme, C.E. Aubin, J.A. De Guise, and J. Dansereau. Longterm three-timensional changes of the spine after posterior spinal instrumentation and fusion in adolescent idiopathic scoliosis. European Spine Journal, 8(1) :16-21, 1999.

[13] S. Delorme, P. Violas, J. Dansereau, J. de Guise, C.-E. Aubin, and H. Labelle. Preoperative and early postoperative three-dimensional changes of the rib cage after posterior instrumentation in adolescent idiopathic scoliosis. European Spine Journal, 10(2) :101$107,2001$.

[14] M. Asher, S. M. Lai, D. Burton, and B. Manna. Maintenance of trunk deformity correction following posterior instrumentation and arthrodesis for idiopathic scoliosis. Spine, 29 :1782-1788, 2004.

[15] R. K. Pratt, J. K. Webb, R. G. Burwell, and A. A. Cole. Changes in surface and radiographic deformity after universal spine system for right thoracic adolescent idiopathic scoliosis : is rib-hump reassertion a mechanical problem of the thoracic cage rather than an effect of relative anterior spinal overgrowth? Spine, 26 :1778-1787, 2001.

[16] U. Willers, E. E. Transfeldt, and R. Hedlund. The segmental effect of cotrel-dubousset instrumentation on vertebral rotation, rib hump and the thoracic cage in idiopathic scoliosis. European Spine Journal, 5(6) :387-393, 1996.

[17] T. R. Haher, A. Merola, R. I. Zipnick, J. Gorup, D. Mannor, and J. Orchowski. Metaanalysis of surgical outcome in adolescent idiopathic scoliosis. a 35-year english literature review of 11,000 patients. Spine, 20(14):1575-1584, 1995.

[18] A. E. Geissele, J. W. Ogilvie, M. Cohen, and D. S. Bradford. Thoracoplasty for the treatment of rib prominence in thoracic scoliosis. Spine, 19(14) :1636-1642, 1994.

[19] K.H. Bridwell. Surgical treatment of idiopathic adolescent scoliosis. Spine, 24(24) :2607-2616, 1999.

[20] C.-E. Aubin, J. Dansereau, F. Parent, H. Labelle, and J. A. de Guise. Morphometric evaluations of personalised $3 \mathrm{~d}$ reconstructions and geometric models of the human spine. Medical and Biological Engineering and Computing, 35(6) :611-618, 1997.

[21] D. Périé, C. E. Aubin, M. Lacroix, Y. Lafon, and H. Labelle. Biomechanical modelling of orthotic treatment of the scoliotic spine including a detailed representation of the brace-torso interface. Medical and Biological Engineering and Computing, 42 :339-344, 2004. 
[22] J. Carrier, C.-E. Aubin, I. Villemure, and H. Labelle. Biomechanical modelling of growth modulation following rib shortening or lengthening in adolescent idiopathic scoliosis. Medical and Biological Engineering and Computing, 42(4) :541-548, 2004.

[23] Clin J., Aubin C.-E., Parent S., Ronsky J., and Labelle H. Biomechanical modeling of brace design. Stud. Health Technol. Inform., 123 :255-260, 2006.

[24] J. Clin, C.-E. Aubin, and H. Labelle. Virtual prototyping of a brace design for the correction of scoliotic deformities. Medical and Biological Engineering and Computing, 45(5):467-473, 2007.

[25] J. Clin, C.-E. Aubin, S. Parent, A. Sangole, and H. Labelle. Comparison of the biomechanical 3D efficiency of different brace designs for the treatment of scoliosis using a finite element model. Eur. Spine J., 19(7) :1169-1178, 2010.

[26] J. Clin, C.-E. Aubin, S. Parent, and H. Labelle. A biomechanical study of the charleston brace for the treatment of scoliosis. Spine, Publish Ahead of Print, 2010.

[27] J. Clin, C.-E. Aubin, H. Labelle, and S. Parent. Immediate correction in brace treatment : how much is needed to obtain a long-term effectiveness? In 8th Meeting of the International Research Society of Spinal Deformities (IRSSD 2010), Montréal, Québec, Canada, 2010.

[28] M. Beauséjour, C.E. Aubin, A.G. Feldman, and H. Labelle. Simulations de tests d'inflexion latérale à l'aide d'un modèle musculo-squelettique du tronc. Annales de chirurgie, $53: 742-750,1999$.

[29] O. Dionne, K. C. Assi, S. Grenier, H. Labelle, F. Guibault, and F. Cheriet. Simulation of the postoperative trunk appearance in scoliotic surgery. In International Symposium On Biomedical Imaging, ISBI 2012, pages 1208-1211, 2012.

[30] U. Meier, O. López, C. Monserrat, M. C. Juan, and M. Alcaniz. Real-time deformable models for surgery simulation : a survey. Computer Methods and Programs Biomedicine, $77(3): 183-197,2005$.

[31] R. M. Koch, M. H. Gross, F. R. Carls, D. F. von Büren, G. Fankhauser, and Y. I. H. Parish. Simulating facial surgery using finite element models. In Proceedings of the 23rd annual conference on Computer graphics and interactive techniques, pages 421428. ACM, 1996. 
[32] R. M. Koch, S. H. M. Roth, M. H. Gross, A. P. Zimmermann, and H. F. Sailor. A framework for facial surgery simulation. In Proceedings of the 18th spring conference on Computer graphics, pages 33-42, 2002.

[33] D. Terzopoulos and K. Waters. Physically-based facial modeling, analysis, and animation. Journal of Visualization and Computer Animation, 1(2) :73-80, 1990.

[34] E. Keeve, S. Girod, and B. Girod. Craniofacial surgery simulation. In Visualization in Biomedical Computing, pages 541-546. Springer : Berlin, 1996.

[35] W. Mollemans, F. Schutyser, J.V. Cleynenbreugel, and P. Suetens. Tetrahedral mass spring model for fast soft tissue deformation. In Surgery Simulation and Soft Tissue Modeling IS4TM 2003, LNCS 2673, pages 145-154. Springer : Berlin, 2003.

[36] M. Bro-Nielsen. Surgery simulation using fast finite elements. In VBC '96 : Proceedings of the 4 th International Conference on Visualization in Biomedical Computing, pages 529-534. Springer-Verlag, 1996.

[37] S. Cotin, H. Delingette, and N. Ayache. Real-time elastic deformations of soft tissues for surgery simulation. IEEE Transactions On Visualization and Computer Graphics, 5(1) :62-73, 1999.

[38] Matthias Müller, Julie Dorsey, Leonard McMillan, Robert Jagnow, and Barbara Cutler. Stable real-time deformations. In SCA '02 : Proceedings of the 2002 ACM SIGGRAPH/Eurographics symposium on Computer animation, pages 49-54. ACM, 2002.

[39] Guillaume Picinbono, Herve Delingette, and Nicholas Ayache. Real-time large displacement elasticity for surgery simulation : Non-linear tensor-mass model. In MICCAI '00 : Proceedings of the Third International Conference on Medical Image Computing and Computer-Assisted Intervention, pages 643-652. Springer-Verlag, 2000.

[40] M. Chabanas, V. Luboz, and Y. Payan. Patient specific finite element model of the face soft tissues for computer-assisted maxillofacial surgery. Medical Image Analysis, $7(2): 131-151,2003$.

[41] Liesbet Roose, Wim De Maerteleire, Wouter Mollemans, and Paul Suetens. Validation of different soft tissue simulation methods for breast augmentation. International Congress Series, 1281 :485-490, 2005.

[42] R. Mahnken and E. Stein. A unified approach for parameter identification of inelastic material models in the frame of the finite element method. Computer Methods in Applied Mechanics and Engineering, 136(3-4) :225 - 258, 1996. 
[43] R. Mahnken and E. Stein. Parameter identification for viscoplastic models based on analytical derivatives of a least-squares functional and stability investigations. International Journal of Plasticity, 12(4) :451 - 479, 1996.

[44] R. Mahnken and E. Stein. Parameter identification for finite deformation elastoplasticity in principal directions. Computer Methods in Applied Mechanics and Engineering, 147(1-2) :17 - 39, 1997.

[45] T. Seibert, J. Lehn, S. Schwan, and F.G. Kollmann. Identification of material parameters for inelastic constitutive models : Stochastic simulations for the analysis of deviations. Continuum Mechanics and Thermodynamics, 12(2) :95 - 120, 2000.

[46] T. Harth, S. Schwan, J. Lehn, and F. G. Kollmann. Identification of material parameters for inelastic constitutive models : statistical analysis and design of experiments. International Journal of Plasticity, 20(8-9) :1403 - 1440, 2004.

[47] W. T. D’Arcy. On Growth and Form. Cambridge University Press, 1917.

[48] U. Grenander and M. I. Miller. Computational anatomy : an emerging discipline. $Q$. Appl. Math., LVI(4) :617-694, 1998.

[49] Daniel P. Huttenlocher, Gregory A. Klanderman, Gregory A. Kl, and William J. Rucklidge. Comparing images using the hausdorff distance. IEEE Transactions on Pattern Analysis and Machine Intelligence, 15 :850-863, 1993.

[50] Guillaume Charpiat, Olivier Faugeras, and Renaud Keriven. Approximations of shape metrics and application to shape warping and empirical shape statistics. Found. Comput. Math., 5(1) :1-58, 2005.

[51] Distance-Based Shape Statistics, volume 5, 2006.

[52] D.G. Kendall. Shape manifolds, procrustean metrics, and complex projective spaces. Bulletin of the London Mathematical Society, 16(2) :81-121, 1984.

[53] F. L. Bookstein. Size and shape spaces for landmark data in two dimensions. Statistical Science, 1(2) :181-242, 1986.

[54] Simone Ceolin, William A. P. Smith, and Edwin Hancock. Facial shape spaces from surface normals and geodesic distance. In DICTA '07 : Proceedings of the 9th Biennial Conference of the Australian Pattern Recognition Society on Digital Image Computing Techniques and Applications, pages 416-423, Washington, DC, USA, 2007. IEEE Computer Society. 
[55] Simone Ceolin, William Smith, and Edwin Hancock. Facial shape spaces from surface normals. In Image Analysis and Recognition, pages 955-965. 2008.

[56] C. R. Rao. Information and accuracy attainable in estimation of statistical parameters. Bulletin of the Calcutta Mathematical Society, 37 :81-91, 1945.

[57] Shape analysis using the Fisher-Rao Riemannian metric : unifying shape representation and deformation, 2006.

[58] Adrian M. Peter and Anand Rangarajan. Information geometry for landmark shape analysis : Unifying shape representation and deformation. IEEE Transactions on Pattern Analysis and Machine Intelligence, 31 :337-350, 2009.

[59] Simone Ceolin and Edwin R. Hancock. Using the fisher-rao metric to compute facial similarity. In ICIAR (1), pages 384-393, 2010.

[60] J. Glaunes, A. Trouvé, and L. Younes. Diffeomorphic matching of distributions : A new approach for unlabelled point-sets and sub-manifolds matching. In In $C V P R$, pages 712-718, 2004.

[61] M. Vaillant and J. Glaunès. Surface matching via currents. In Proceedings of Information Processing in Medical Imaging (IPMI 2005), number 3565 in Lecture Notes in Computer Science, pages 381-392, 2005.

[62] J. Glaunès, A. Qiu, M. I. Miller, and L. Younes. Large deformation diffeomorphic metric curve mapping. Int. J. Comput. Vision, 80(3) :317-336, 2008.

[63] S. Durrleman, X. Pennec, A. Trouvé, and N. Ayache. Statistical models of sets of curves and surfaces based on currents. Medical Image Analysis, 13(5) :793-808, 2009. Includes Special Section on the 12th International Conference on Medical Imaging and Computer Assisted Intervention.

[64] N. Acir and C. Guzelis. Automatic spike detection in eeg by a two-stage procedure based on support vector machines. Computers in Biology and Medicine, 34(7) :561$575,2004$.

[65] G.-Z. Li, J. Yang, C.-Z. Ye, and D.-Y. Geng. Degree prediction of malignancy in brain glioma using support vector machines. Comput. Biol. Med., 36(3) :313-325, 2006.

[66] C. Bergeron, F. Cheriet, J. Ronsky, R. Zernicke, and H. Labelle. Prediction of anterior scoliotic spinal curve from trunk surface using support vector regression. Eng. Appl. Artificial Intell., 18(8):973-983, 2005. 
[67] J. Jaremko, P. Poncet, J. Ronsky, J. Harder, J. Harder, J. Dansereau, H. Labelle, and R. Zernicke. Genetic algorithm-neural network estimation of cobb angle from torso asymmetry in scoliosis. J. Biomech. Eng., 124(5) :496-503, 2002.

[68] L. Ramirez, N.G. Durdle, V.J. Raso, and D.L. Hill. A support vector machines classifier to assess the severity of idiopathic scoliosis from surface topography. IEEE Trans. Inf. Technol. Biomed., 10(1) :84-91, 2006.

[69] L. Seoud, M.M. Adankon, H. Labelle, J. Dansereau, and F. Cheriet. Prediction of scoliosis curve type based on the analysis of trunk surface topography. In Biomedical Imaging : From Nano to Macro, 2010 IEEE International Symposium on, pages 408411, 2010.

[70] T.M. Cover and P.E. Hart. Nearest neighbor pattern classification. IEEE Transactions on Information Theory, IT-13 :21-27, 1967.

[71] T. M. Cover. Estimation by the nearest neighbor rule. IEEE Transactions on Information Theory, IT-14(1) :50-55, 1968.

[72] L. Devroye, L. Gyorfi, A. Krzyzak, and G. Lugosi. On the strong universal consistency of nearest neighbor regression function estimates. The Annals of Statistics, 22 :13711385, 1994.

[73] S. R. Kulkarni and S. E. Posner. Rates of convergence of nearest neighbor estimation under arbitrary sampling. IEEE Trans. Inf. Theory, 41 :1028-1039, 1995.

[74] G. Biau, F. Cérou, and A. Guyader. Rates of convergence of the functional k-nearest neighbor estimate. IEEE Transactions on Information Theory, 56 :2034-2040, 2010.

[75] Kilian Weinberger, John Blitzer, and Lawrence Saul. Distance metric learning for large margin nearest neighbor classification. In Y. Weiss, B. Schölkopf, and J. Platt, editors, Advances in Neural Information Processing Systems 18, pages 1473-1480. MIT Press, Cambridge, MA, 2006.

[76] K. Weinberger and L. Saul. Fast solvers and efficient implementations for distance metric learning. pages 1160-1167, 2008.

[77] K.Q. Weinberger and L.K. Saul. Distance metric learning for large margin nearest neighbor classification. Journal of Machine Learning Research, 10.

[78] C. Domeniconi, J. Peng, and D. Gunopulos. Locally adaptive metric nearest neighbor classification. IEEE Trans. Pattern Anal. Mach. Intell., 24(9) :1281-1285, 2002. 
[79] T. Hastie and R. Tibshirani. Discriminant adaptive nearest neighbor classification. IEEE Trans. Pattern Anal. Mach. Intell., 18(6) :607-615, 1996.

[80] Y. Lee, D. Terzopoulos, and K. Waters. Realistic modeling for facial animation. In SIGGRAPH '95 : Proceedings of the 22nd annual conference on Computer graphics and interactive techniques, pages 55-62, New York, NY, USA, 1995. ACM.

[81] S. Sarni, A. Maciel, R. Boulic, and D. Thalmann. Evaluation and visualization of stress and strain on soft biological tissues in contact. In SMI '04 : Proceedings of the Shape Modeling International 2004, pages 255-262, Washington, DC, USA, 2004. IEEE Computer Society.

[82] S. Delorme, Y. Petit, J. A. de Guise, H. Labelle, C.-E. Aubin, and J. Dansereau. Assessment of the 3D reconstruction and high-resolution geometrical modeling of the human skeletal trunk from 2D radiographic images. IEEE Transactions on Biomedical Engineering, 50(8) :989-98, 2003.

[83] H. Si. Tetgen : A quality tetrahedral mesh generator and three-dimensional delaunay triangulator. http ://tetgen.berlios.de/.

[84] J. R. Shewchuk. Tetrahedral mesh generation by delaunay refinement. In SCG '98 : Proceedings of the fourteenth annual symposium on Computational geometry, pages 86-95. ACM, 1998.

[85] M. Teschner, B. Heidelberger, M. Müller, and M. Gross. A versatile and robust model for geometrically complex deformable solids. In Proceedings of the Computer Graphics International, pages 312-319. IEEE Computer Society, 2004.

[86] J. L. Jaremko, P. Poncet, J. Ronsky, J. Harder, and J. Dansereau. Indices of torso asymmetry related to spinal deformity in scoliosis. Clinical Biomechanics, 17(8) :559$568,2002$.

[87] L. Seoud, F. Cheriet, H. Labelle, and J. Dansereau. A novel method for the 3d reconstruction of scoliotic ribs from frontal and lateral radiographs. IEEE Transactions in biomedical engineering, 58(5) :1135-1146, 2011.

[88] V. Pazos, F. Cheriet, J. Dansereau, Janet Ronsky, Ronald F. Zernicke, and Hubert Labelle. Reliability of trunk shape measurements based on 3-d surface reconstructions. European Spine Journal, 16(11) :1882-1891, 2007. 
[89] R. Buchanan, J. G. Birch, A. A. Morton, and R. H. Browne. Do you see what I see? Looking at scoliosis surgical outcomes through orthopedists' eyes. Spine, 28(24) :2700$2704,2003$.

[90] Goran Devedzic, Sasa Cukovic, Vanja Lukovic, Danijela Milosevic, K. Subburaj, and Tanja Lukovic. Scoliomedis : Web-oriented information system for idiopathic scoliosis visualization and monitoring. Computer Methods and Programs in Biomedicine, 108(2) :736-749, 2012.

[91] V. Pazos, F. Cheriet, H. Labelle, and J. Dansereau. 3d reconstruction and analysis of the whole trunk surface for non-invasive follow-up of scoliotic deformities. Studies in health technology and informatics, 91 :296-299, 2002.

[92] T.M.L. Shannon. Dynamic Surface Topography and Its Application to the Evaluation of Adolescent Idiopathic Scoliosis. PhD thesis, Oxford Brookes University, Oxford, U.K., september 2010.

[93] Peter O. Ajemba, Nelson G. Durdle, and V. James Raso. Characterizing torso shape deformity in scoliosis using structured splines models. IEEE Trans. Biomed. Engineering, 56(6) :1652-1662, 2009.

[94] S. Li, W. Zhou, Q. Yuan, S. Geng, and D. Cai. Feature extraction and recognition of ictal eeg using emd and svm. Computers in Biology and Medicine, 43(7) :807-816, 2013 .

[95] A. Subasi. Classification of emg signals using pso optimized svm for diagnosis of neuromusculardisorders. Computers in Biology and Medicine, 43(5) :576-586, 2013.

[96] E. Comak, A. Arslan, and I. Türkoglu. A decision support system based on support vector machines for diagnosis of the heart valve diseases. Computers in biology and Medicine, 37(1) :21-27, 2007.

[97] S. Kiranyaz, T. Ince, J. Pulkkinen, M. Gabbouj, J. Ärje, S. Kärkkäinen, V. Tirronen, M. Juhola, T. Turpeinen, and K. Meissner. Classification and retrieval on macroinvertebrate image databases. Computers in Biology and Medicine, 41(7) :463-472, 2011.

[98] H. M. Muda, P. Saad, and R. M. Othman. Remote protein homology detection and fold recognition using two-layer support vector machine classifiers. Computers in Biology and Medicine, 41(8):687-699, 2011.

[99] T. Hastie, R. Tibshirani, and Friedman. The elements of statistical learning. 
[100] H. Hotelling. Analysis of a complex of statistical variables into principal components. Journal of Educational Psychology, 24 :417-441, 1933.

[101] B. Schölkopf, A.J. Smola, and K.-R. Müller. Nonlinear component analysis as a kernel eigenvalue problem. Neural Computation, 10(5) :1299-1319, 1998.

[102] X. He and P. Niyogi. Locality Preserving Projections. Cambridge, MA, 2004. MIT Press.

[103] X. He, D. Cai, S. Yan, and H.-J. Zhang. Neighborhood preserving embedding. In Proceedings of IEEE International Conference on Computer Vision, volume 2, pages 1208-1213. IEEE, 2005.

[104] H. Wold. Soft Modeling by Latent Variables; the Nonlinear Iterative Partial Least Squares Approach. Perspectives in Probability and Statistics. Papers in Honour of M. S. Bartlett, 1975.

[105] S. Wold, H. Ruhe, H. Wold, and W.J. Dunn III. The collinearity problem in linear regression. the partial least squares (PLS) approach to generalized inverse. SIAM Journal of Scientific and Statistical Computations, 5(3) :735-743, 1984.

[106] V. Pazos, F. Cheriet, L. Song, H. Labelle, and J. Dansereau. Accuracy assessment of human trunk surface 3d reconstructions from an optical digitizing system. Medical and Biological Engineering and Computing, 43(1) :11-15, 2005.

[107] J.A.K. Suykens, V.T. Gestel, J. De Brabanter, B. De Moor, and J. Vandewalle. Least Squares Support Vector Machines. World Scientific, Singapore, 2002.

[108] S.-M. Huang and J.-F. Yang. Unitary regression classification with total minimum projection error for face recognition. IEEE signal processing letters, 20(5) :443-446, 2013.

[109] B.L. Pellom, R. Sarikaya, and J.H.L. Hansen. Fast likelihood computation techniques in nearest-neighbor based search for continuous speech recognition. IEEE Signal Processing Letters, 8(8) :221-224, 2001.

[110] S. Marano, V. Matta, and P. Willett. Nearest-neighbor distributed learning by ordered transmissions. IEEE Transactions on Signal Processing, 61(21) :221-224, 2013.

[111] Dor Kedem, Stephen Tyree, Kilian Weinberger, Fei Sha, and Gert Lanckriet. Non-linear metric learning. In P. Bartlett, F.C.N. Pereira, C.J.C. Burges, L. Bottou, and K.Q. 
Weinberger, editors, Advances in Neural Information Processing Systems 25, pages 2582-2590. 2012.

[112] B. Kulis. Metric learning : A survey. Foundations and Trends in Machine Learning, 5(4) :287-364, 2013.

[113] J. Devore and N. Farnum. Applied Statistics for Engineers and Scientists. Duxbury Press, 1999.

[114] C.-É. Aubin, Y. Petit, I.A.F. Stokes, F. Poulin, M. Gardner-Morse, and H. Labelle. Biomechanical modeling of posterior instrumentation of the scoliotic spine. Computer Methods in Biomechanics and Biomedical Engineering, 6(1) :27-32, 2003.

[115] S. Cotin, H. Delingette, and N. Ayache. A hybrid elastic model allowing real-time cutting, deformations and force-feedback for surgery training and simulation. The Visual Computer, 16 :437-452, 2000.

[116] R. Harmouche, F. Cheriet, H. Labelle, and J. Dansereau. 3D registration of MR and X-ray spine images using an articulated model. Computerized Medical Imaging and Graphics, 36(5) :410 - 418, 2012.

[117] K.M Cheung and K.D Luk. Prediction of correction of scoliosis with se of the fulcrum bending radiograph. J. Bone Joint Surg. Am., 79 :1144-1150, 1997.

[118] P. Debanné, V. Pazos, H. Labelle, and F. Cheriet. Evaluation of reducibility of trunk asymmetry in lateral bending. In 8th Meeting of the International Research Society of Spinal Deformities (IRSSD 2010), Montréal, Québec, Canada, 2010. 